\title{
The static quark potential in $2+1$ flavour Domain Wall QCD from QCDOC
}

\author{
Koichi Hashimoto* \\ Institute for Theoretical Physics, Kanazawa University, Kanazawa 920-1192, Japan \\ Radiation Laboratory, RIKEN, Wako 351-0198, Japan \\ E-mail: koichi@hep.s.kanazawa-u.ac.jp
}

\section{Taku Izubuchi}

Institute for Theoretical Physics, Kanazawa University, Kanazawa 920-1192, Japan

RIKEN-BNL Research Center, Brookhaven National Laboratory, Upton, New York 11973, USA

E-mail: izubuchi@hep.s.kanazawa-u.ac.jp

\section{Jun Noaki}

School of Physics and Astronomy, University of Southampton, Southampton SO17 1ST, England

E-mail: noaki@phys.soton.ac.uk

\section{RBC and UKQCD Collaborations}

\begin{abstract}
We report our present status of on-going project on the measurement of the static quark potential in 2+1 flavour domain wall QCD with various improved gauge actions and couplings. Lattice spacing determined from Sommer scale on these ensembles are from $1.6 \mathrm{GeV}$ to $2.0 \mathrm{GeV}$ for $16^{3} \times 32$ lattice with fifth dimension size 8 . We also examine size of discretization error from scaling of a pair of dimensionless quantities, $\left(r_{0} m_{\pi}\right)^{2}$ and $r_{0} m_{\rho}$, and found small scaling violation.
\end{abstract}

XXIIIrd International Symposium on Lattice Field Theory

25-30 July 2005

Trinity College, Dublin, Ireland

\footnotetext{
* Speaker.
} 


\section{Introduction}

The only way to remove pending issues associated with systematic errors due to quenching in lattice QCD is carrying out simulation with dynamical quarks. Among several ongoing projects generating 2+1 flavour dynamical gauge ensembles, a striking characteristics of that by the RBCUKQCD Collaborations is in their usage of domain wall fermions (DWF) for the sea quarks. DWF breaks chiral symmetry only by a small amount and thus has least discretization error. There are a lot of extensive measurements planned on the ensemble in near future.

We report our calculation of the static quark potential and preliminary results of lattice spacing $a$ from Sommer scale $r_{0} \approx 0.5 \mathrm{fm}[1]$ on the RBC-UKQCD gauge ensembles.

\section{Calculation}

The static potential $V(\vec{r})$ between a quark and anti-quark pair at relative spatial displacement $\vec{r}$, is calculated from the Wilson loop $\langle W(\vec{r}, t)\rangle:\langle W(\vec{r}, t)\rangle=C(\vec{r}) e^{-V(\vec{r}) t}+$ ("excited states"), with a normalisation $C(\vec{r}=\overrightarrow{0})=1$.

We calculate $\langle W(\vec{r}, t)\rangle$ in lattice QCD with 2+1 flavour domain wall fermion [2] and several gauge actions (Iwasaki [3], DBW2 [4], and more negative rectangle coefficient, $c_{1}$, actions) on $16^{3} \times 32$ lattice with fifth dimension size, $L_{s}=8$, and domain wall height, $M_{5}=1.8$. The reason for calculating several gauge actions was to search for actions producing smaller residual mass, $m_{\text {res }}$, at fixed $L_{s}$ and lattice spacing $a$ [5]. In quenched simulations, changing $c_{1}$ caused a drastic reduction of $m_{\mathrm{res}}: m_{\mathrm{res}}^{(\text {Wilson) }} \approx 10 \times m_{\mathrm{res}}^{(\text {Iwasaki) }} \approx 100 \times m_{\mathrm{res}}^{(\mathrm{DBW} 2)}$ at $a^{-1} \approx 2 \mathrm{GeV}$ [6]. We use three degenerate up and down quark masses, $m_{u, d}=0.01,0.02,0.04$, and the strange quark mass $m_{s}=0.04$ was our best pre-simulation estimate of the physical strange quark mass. Simulation parameters are tabled in Table 1,2 and 3

We compare two independent analyses, both of which implement APE smearing [7] for spatial links to improve the signal/noise.

The main difference between these analyses is the path connecting the quark and anti-quark in a time slice (Figure 11). The first analysis (Type-I) is carried out using the closest path to the diagonal line connecting the quark-antiquark. This path is determined by Bresenham algorithm [8] in the same way as [9,10]. The second path (Type-II) is the taxi driver's path employed by Chroma code [11]. The smearing coefficient and number of smearing steps are tuned to be $\left(c_{\text {smear }}, N_{\text {smear }}\right)=$ $(0.50,20 \sim 40)$, where the overlap with ground state, $C(\vec{r})$, is approximately its maximum.

The physical parameters are obtained by fitting the lattice value of $V(\vec{r})$ to the function: $V(\vec{r})=$ $V_{0}-\alpha /|\vec{r}|+\sigma|\vec{r}|$, from which Sommer scale $r_{0} / a=\sqrt{(1.65-\alpha) / \sigma}$ is determined in lattice unit. For each gauge action and fixed gauge coupling $\beta$, we estimate lattice scale $a^{-1}$ assuming $r_{0}=0.5$ $\mathrm{fm}$ in the chiral limit. Note this assumption turns out to be consistent with the other scale setting ansatz using rho meson mass, $m_{\rho}=770 \mathrm{MeV}$ in previous simulations [9, 12].

\section{Results}

Figure 2 shows potentials measured using Type-I and Type-II paths. These two analyses were performed on same ensemble ( $\beta=2.2, m_{u, d}=m_{s}=0.04$ on Table 1 , and central values are consistent with each other within statistical error. Sharply impoved signals (red circles) for relatively 


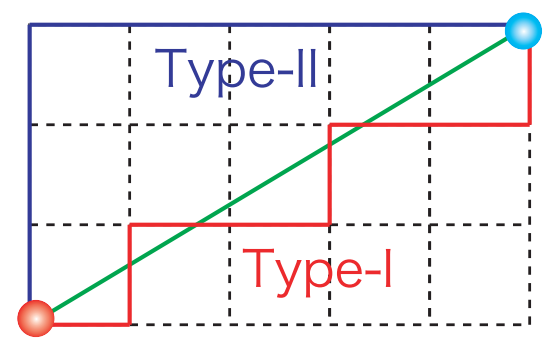

Figure 1: Sketch of the path connecting quark and anti-quark. Type-I (red) is the closest path to diagonal line (green) by Bresenham algorithm, and Type-II (blue) is the taxi driver's path.

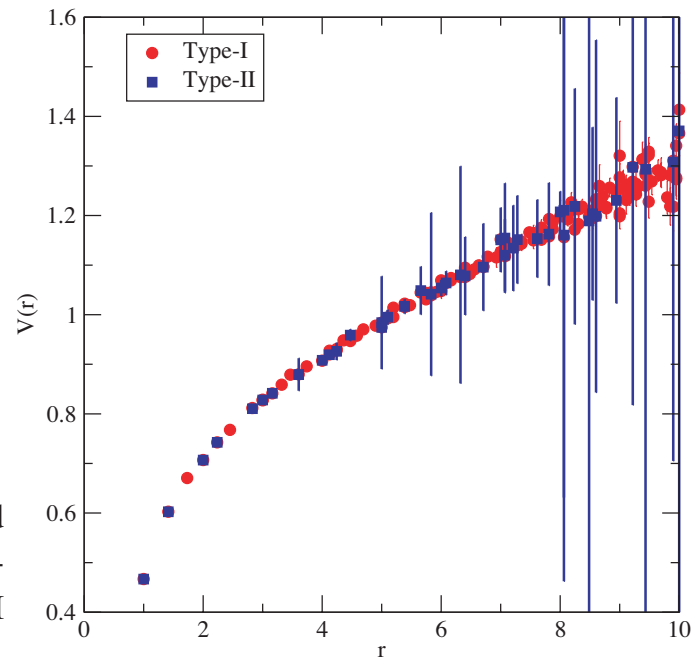

Figure 2: $V(\vec{r})$ vs $|\vec{r}|$ for Iwasaki action, $\beta=2.20$, $m_{u, d}=m_{s}=0.04$.

long distance off-axis spatial paths indicate that the $\bar{Q}-Q$ state by Type-I has larger overlap with the ground state than that by Type-II. This is reasonably consistent with the picture of the QCD-string connecting quark and anti-quark.

The time separation of Wilson loop, $t$, is chosen to minimise excited states contamination to the ground state potential by monitoring $t$ dependence of $V(\vec{r})$. Then $V(\vec{r})$ is fit to the formula for $|\vec{r}| \in$ $\left[r_{\min }, r_{\text {max }}\right]$. Figure 3 show $r_{0}$ as a function of dynamical quark mass, $m_{u, d}+m_{\text {res }}$, for several fixed $\beta$ 's. To estimate lattice spacing $a$, we take the extrapolation to the point $m_{u, d}+m_{\text {res }}=0$ linearly and obtain $r_{0} / a$ in this limit (open symbols) from simulation points (filled symbols). Preliminary results of this extrapolation and the value of $a^{-1}$ are summarised in Table 1,2 and 3 in which data at the simulation points are listed as well. For the Type-I data, we estimate systematic errors by the dependence of the results on the fit ranges in extracting potentials from Wilson loop and in fitting the potentials to the specific function introduced in Section 1. We estimate these errors from the variation of central values for $r_{\text {min }} \in[\sqrt{2}, \sqrt{6}], r_{\max } \in[7,9], t=5,6$. Available results for Iwasaki $\beta=2.13$ and DBW2 $\beta=0.764$ are obtained by Type-II path. Analysis by Type-I path is under progress. In particular, RBC-UKQCD has started their major production run using Iwasaki $\beta=2.13$ on $24^{3} \times 64$ lattice with $L_{s}=16$. Our results for Iwasaki $\beta=2.13$ provide properties of this ensemble.

Finally we measure the magnitude of discretization error using the data from all ensembles we measured. Due to the (almost exact) chiral symmetry of DWF, the scaling violation is expected as $\mathscr{O}\left(\left(a \Lambda_{Q C D}\right)^{2}\right)+\mathscr{O}\left(a m_{\text {res }}\right) \sim \mathscr{O}(1) \%$ for the parameters used. We examine this expectation by plotting a pair of dimensionless quantities, $\left(\left(r_{0} m_{\pi}\right)^{2}, r_{0} m_{\rho}\right)$ in Figure $4 m_{\pi}, m_{\rho}$ are pseudo-scalar and vector meson masses respectively at relatively large valence quark mass set to be equal to sea quark mass $m_{u, d}$ [13]. If scaling were perfect (no discretization error), all data points are on a universal curve in the two dimensional plane. The left panel of Figure 4 demonstrates the expectation is true for these quantities: points are on a line within statistical error $(\lesssim 5 \%)$.

We also compare DBW2 $\beta=0.80$ results with those of two flavour (red square) and quenched 

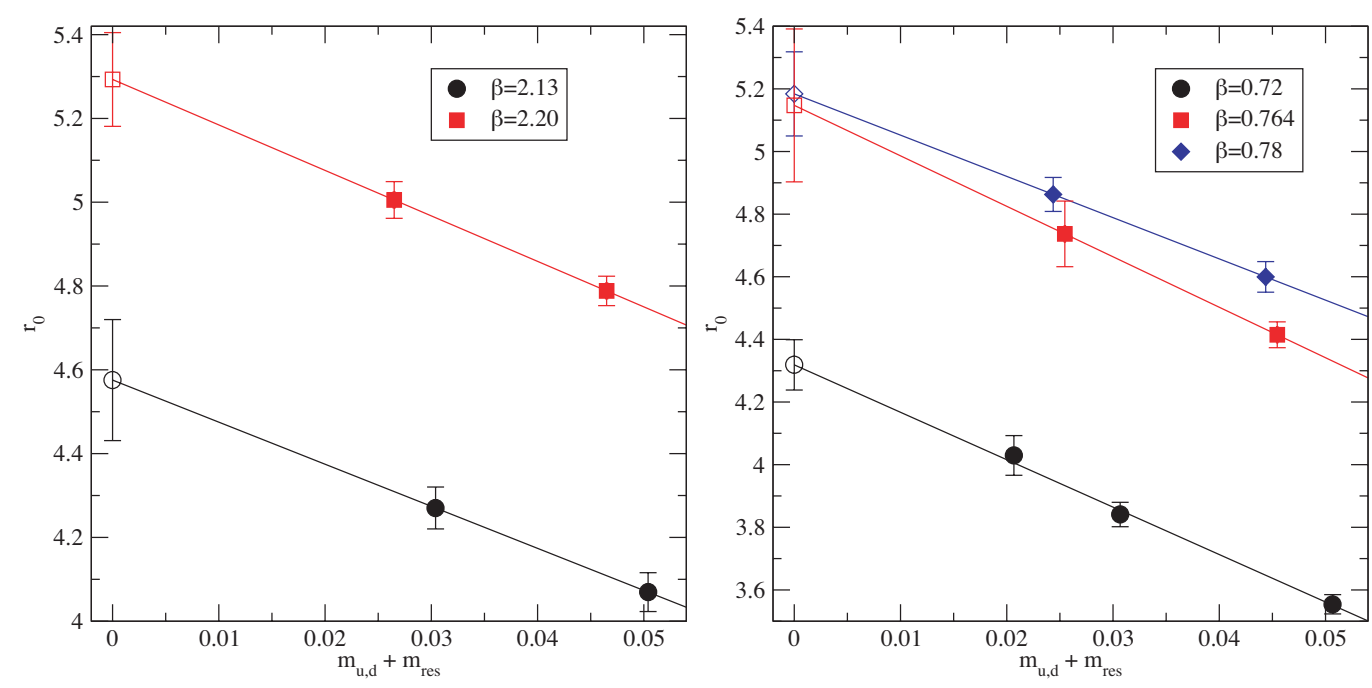

Figure 3: $r_{0}$ as a function of dynamical quark mass for Iwasaki (left) and DBW2 (right) gauge action. Each line shows linear extrapolation to the chiral limit, $m_{u, d}+m_{\mathrm{res}} \rightarrow 0$, for fixed $\beta$ 's indicated in legends.

\begin{tabular}{|c|c|c|c|c|c|c|}
\hline$\beta$ & $m_{u, d}$ & \# conf & Alg & Path & $r_{0} / a$ & $a^{-1}[\mathrm{GeV}]$ \\
\hline \multirow{3}{*}{2.20} & 0.04 & 320 & & & $4.788(35)(30)$ & $1.890(14)(12)$ \\
& 0.02 & 177 & RHMC & Type-I & $5.005(44)(48)$ & $1.975(17)(19)$ \\
& $-m_{\text {res }}=-0.0065(1)$ & - & & & $5.293(112)(81)$ & $2.089(44)(32)$ \\
\hline \multirow{3}{*}{2.13} & 0.04 & 306 & & & $4.069(46)$ & $1.606(18)$ \\
& 0.02 & 300 & \multirow{2}{*}{ RHMC } & Type-II & $4.270(50)$ & $1.685(20)$ \\
& $-m_{\text {res }}=-0.0104(2)$ & - & & & $4.576(145)$ & $1.806(57)$ \\
\hline
\end{tabular}

Table 1: Results of $r_{0}$ for Iwasaki gauge action $\left(c_{1}=-0.331\right) . m_{s}=0.04, L_{s}=8, M_{5}=1.8$. Note our estimation for lattice cut off, $a^{-1}$, is the values at chiral limit, $m_{u, d} \rightarrow-m_{\text {res }}$.

\begin{tabular}{|c|c|c|c|c|c|c|}
\hline$\beta$ & $m_{u, d}$ & \# conf & Alg & Path & $r_{0} / a$ & $a^{-1}[\mathrm{GeV}]$ \\
\hline 0.80 & 0.04 & 200 & Ralg & $\begin{array}{c}\text { Type } \\
\text {-I }\end{array}$ & $5.014(38)(67)$ & $1.979(15)(26)$ \\
& 0.04 & 165 & & & $4.600(49)(16)$ & $1.815(19)(6)$ \\
\hline \multirow{3}{*}{0.78} & 0.02 & 180 & RHMC & Type & $4.863(54)(142)$ & $1.929(21)(56)$ \\
& $-m_{\text {res }}=-0.00437(6)$ & - & & - I & $5.184(134)(335)$ & $2.046(53)(132)$ \\
\hline \multirow{3}{*}{0.764} & 0.04 & 310 & & & $4.415(41)$ & $1.742(16)$ \\
& 0.02 & 300 & \multirow{2}{*}{ RHMC } & Type & $4.737(105)$ & $1.869(41)$ \\
& $-m_{\text {res }}=-0.00546(7)$ & - & & $-I I$ & $5.147(244)$ & $2.031(96)$ \\
\hline \multirow{3}{*}{0.72} & 0.04 & 280 & & & $3.554(31)(67)$ & $1.403(12)(27)$ \\
& 0.02 & 400 & \multirow{2}{*}{ RHMC } & Type & $3.841(39)(61)$ & $1.516(15)(24)$ \\
& 0.01 & 150 & & $-\mathrm{I}$ & $4.029(63)(27)$ & $1.590(25)(11)$ \\
& $-m_{\text {res }}=-0.01066(7)$ & - & & & $4.319(80)(28)$ & $1.704(32)(11)$ \\
\hline
\end{tabular}

Table 2: Same as Table 1 for DBW2 action $\left(c_{1}=-1.4069\right)$. 


\begin{tabular}{|c|c|c|c|c|c|c|}
\hline$\beta$ & $c_{1}$ & \# conf & Alg & Path & $r_{0} / a$ & $a^{-1}[\mathrm{GeV}]$ \\
\hline 0.53 & -2.3 & 160 & Ralg & Type-I & $5.074(48)(48)$ & $2.002(19)(19)$ \\
0.48 & & 180 & & & $4.002(57)(60)$ & $1.579(22)(24)$ \\
\hline 0.36 & & 135 & & & $5.060(66)(33)$ & $1.997(26)(13)$ \\
0.33 & -3.57 & 145 & Ralg & Type-I & $4.335(47)(102)$ & $1.711(19)(40)$ \\
0.32 & & 180 & & & $3.968(49)(43)$ & $1.566(19)(17)$ \\
\hline 0.16 & -7.47 & 165 & Ralg & Type-I & $4.050(57)(43)$ & $1.598(22)(17)$ \\
\hline
\end{tabular}

Table 3: Results of $r_{0} / a$ and corresponding lattice scale $a^{-1}$ from the simulations with various $c_{1}$ 's with $m_{u, d}=m_{s}=0.04$.
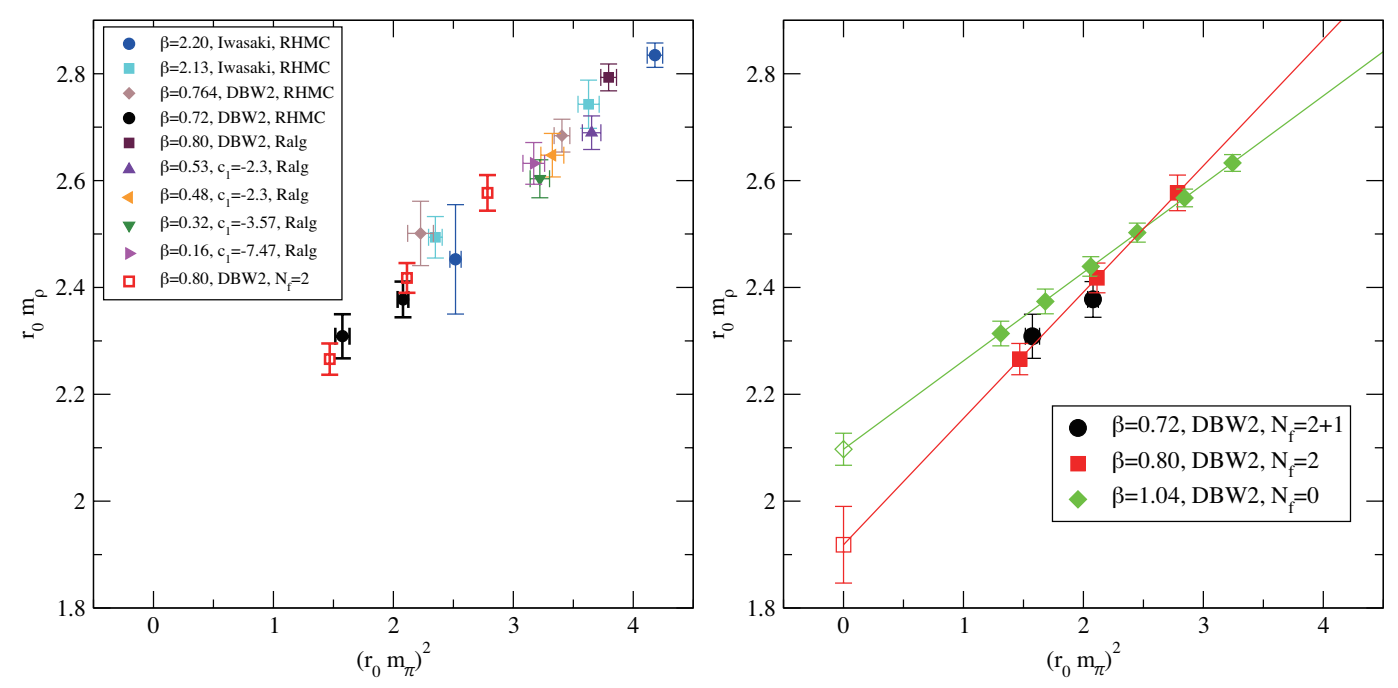

Figure 4: $r_{0} m_{\rho}$ vs. $\left(r_{0} m_{\pi}\right)^{2}$ for $N_{f}=2+1$ with $m_{s}=0.04$ (left). Right panel is the same plot for DBW2 $N_{f}=0(\beta=1.04), N_{f}=2(\beta=0.80)$, and $N_{f}=2+1\left(\beta=0.72, m_{s}=0.04\right)$ with linear chiral extrapolation $\left(r_{0} m_{\pi}\right)^{2} \rightarrow 0$ for $N_{f}=2$ and 0 . Error is uncorrelated between $r_{0}$ and meson spectra.

(green diamonds) simulation in the right panel. The universal line of two flavour points roughly accommodate the $2+1$ flavour points. It is expected because $m_{s}$ is heavy and has less effect on up and down quark meson masses than $u$ and $d$ quarks. On the other hand, quenched data have significantly smaller slope than other two data sets leading larger value of $r_{0} m_{\rho}$ in the chiral limit.

However, we note that the estimation of statistical errors are not so accurate that they reflect the actuality completely since the length of total trajectory and the separation between measured trajectories may not be large enough for some of ensembles.

\section{Acknowledgement}

We thank Peter Boyle, Mike Clark, Saul Cohen, Sam Li, Meifeng Lin, Chris Maynard, and Robert Tweedie, and Azusa Yamaguchi for generating ensembles and meson masses used in this work. We thank Peter Boyle, Dong Chen, Norman Christ, Mike Clark, Saul Cohen, Calin Cristian, Zhihua Dong, Alan Gara, Andrew Jackson, Balint Joo, Chulwoo Jung, Richard Kenway, 
Changhoan Kim, Ludmila Levkova, Xiaodong Liao, Guofeng Liu, Robert Mawhinney, Shigemi Ohta, Konstantin Petrov, Tilo Wettig and Azusa Yamaguchi for developing with us the QCDOC machine and its software. This development and the resulting computer equipment used in this calculation were funded by the U.S. DOE grant DE-FG02-92ER40699, PPARC JIF grant PPA/J/S/1998/00756 and by RIKEN. This work was supported by DOE grant DE-FG02-92ER40699 and we thank RIKEN, BNL, the U.S. DOE and University of Edinburgh for providing the facilities essential for the completion of this work. We also thank the RIKEN Super Combined Cluster (RSCC) at RIKEN, for the computer resources used for the static quark potential calculation. K.H. thanks RIKEN BNL Research Center for its hospitality where this work was performed.

\section{References}

[1] R. Sommer, "A New Way to Set the Energy Scale in Lattice Gauge Theories and its Application to the Static Force and $\alpha_{s}$ in SU(2) Yang-Mills Theory", Nucl. Phys. B411 (1994) 839,

[hep-lat/9310022].

[2] D. B. Kaplan, "A Method for Simulating Chiral Fermions on the Lattice”, Phys. Lett. B288 (1992) 342, [hep-lat/9206013] ; Y. Shamir, "Chiral Fermions from Lattice Boundaries", Nucl. Phys. B406 (1993) 90, [hep-lat/9303005 ] ; V. Furman, Y. Shamir, "Axial symmetries in lattice QCD with Kaplan fermions", Nucl. Phys. B439 (1995) 54 [hep-lat/9405004] .

[3] Y. Iwasaki (1983), "Renormalization Group Analysis of Lattice Theories and Improved Lattice Action", uTHEP-117, uTHEP-118; Y. Iwasaki and T. Yoshie, "Renormalization group improved action for SU (3) lattice gauge theory and the string tension", Phys. Lett. B143 (1984) 449.

[4] T. Takaishi, "Heavy quark potential and effective actions on blocked configurations", Phys. Rev. D54 (1996) 1050; P. de Forcrand et al. (QCD-TARO), "Renormalization group flow of SU(3) lattice gauge theory - Numerical studies in a two coupling space”, Nucl. Phys. B577 (2000) 263 [hep-lat/9911033].

[5] R. D. Mawhinney (RBC and UKQCD) in these proceedings.

[6] Y. Aoki et al. (RBC), "Domain wall fermions with improved gauge actions”, Phys. Rev. D69 (2004) 074504, [hep/lat-0211023].

[7] M. Albanese et al. (APE), “Glueball masses and String tension in Lattice QCD”, Phys. Lett. B192 (1987) 163.

[8] B. Bolder et al., "A High Precision Study of the QQ Potential from Wilson Loops in the Regime of String Breaking”, Phys. Rev. D63 (2001) 074504, [hep-lat / 0411006 ] .

[9] Y. Aoki et al. (RBC), "Lattice QCD with two dynamical flavors of domain wall quarks", hep-lat/0411006.

[10] K. Hashimoto and T. Izubuchi (RBC), "Static $\bar{Q}-Q$ Potential from $N_{f}=2$ Dynamical Domain-Wall QCD”, Nucl. Phys B140 (Proc. Suppl.) (2005) 341, [hep-lat/ 0409101 ] .

[11] R. G. Edwards and B. Joo, "The Chroma Software System for Lattice QCD”, Nucl. Phys B140 (Proc. Suppl.) (2005) 832, [hep-lat/ 0409003 ].

[12] T. Izubuchi (RBC), "Hadron Spectrum and Decay Constant from $N_{F}=2$ Domain Wall QCD”, Nucl. Phys B140 (Proc. Suppl.) (2005) 237, [hep-lat/ 0410034 ].

[13] M. Lin (RBC and UKQCD) in these proceedings; C. M. Maynard (RBC and UKQCD) in these proceedings; R. Tweedie (RBC and UKQCD) in these proceedings. 